\title{
Antitumor effects of imatinib mesylate and synergistic cytotoxicity with an arsenic compound in neuroblastoma cell lines
}

\author{
KYUNG-JIN KIM ${ }^{1}$, JUNG-MOON JUNG ${ }^{1}$, JUNG-YOUN CHO ${ }^{1}$, SO-YOUN WOO ${ }^{1}$, \\ KYUNG-AH CHO ${ }^{1}$, KYUNG-HA RYU ${ }^{2}$ and EUN-SUN YOO ${ }^{2}$ \\ Departments of ${ }^{1}$ Microbiology, and ${ }^{2}$ Pediatrics, School of Medicine, Ewha Womans University, Seoul, Republic of Korea
}

Received December 8, 2010; Accepted February 24, 2011

DOI: 10.3892/etm.2011.220

\begin{abstract}
Neuroblastoma is a common tumor in childhood and exhibits heterogeneity and malignant progression. MYCN expression and amplification profiles are frequently correlated with the efficacy of therapy. Arsenic trioxide and imatinib mesylate (STI-571) have been suggested as promising therapeutic agents for neuroblastoma, which has been shown to be resistant to conventional therapy. In order to ascertain whether the combination of arsenic trioxide and STI-571 exerts a synergistic cytotoxic effect on neuroblastoma cells in relation to MYCN status, we evaluated cellular proliferation after $72 \mathrm{~h}$ of exposure to arsenic trioxide and STI-571 with or without siRNA against MYCN in SH-SY5Y, SK-N-SH and SK-N-BE(2) neuroblastoma cells. Arsenic trioxide and STI-571 demonstrated a synergistic inhibitory effect on cellular proliferation, while MYCN knockdown had an antagonistic effect on this combined treatment. These results indicate that STI-571 treatment may prove effective for MYCN-expressing or MYCN-amplified neuroblastoma. Furthermore, siRNA therapy targeted to MYCN should be avoided in combination with STI-571 treatment in cases of neuroblastoma.
\end{abstract}

\section{Introduction}

Neuroblastoma is the most common extracranial solid tumor in childhood and has been associated with marked clinical, histological and genetic heterogeneity. Most often, the progression of the clinical course results in a poor response to conventional treatment. The prognosis of neuroblastoma depends on the genetic alterations of the cancer cells, which may include non-random deletions, rearrangements or amplification within the chromosome. Among these alterations, the most influential appears to be an MYCN amplification. Since more than

Correspondence to: Dr Eun-Sun Yoo, Department of Pediatrics, School of Medicine, Ewha Womans University, Seoul 158-710, Republic of Korea

E-mail: eunsyoo@ewha.ac.kr

Key words: imatinib mesylate (STI-571), arsenic trioxide, neuroblastoma cells, MYCN
$60 \%$ of patients die from aggressive disease progression, it is critical to develop effective therapeutic agents for the treatment of neuroblastoma, regardless of the heterogeneous nature and genetic alterations involved.

Arsenic trioxide $\left(\mathrm{As}_{2} \mathrm{O}_{3}\right)$ has been shown to exert a cytotoxic effect on a variety of tumors, including acute promyelocytic leukemia, multiple myeloma, esophageal carcinoma and neuroblastoma, regardless of whether MYCN is amplified (1). Arsenic trioxide induces apoptosis via the generation of reactive oxygen species, the disruption of mitochondrial transmembrane potential $(\Delta \Psi \mathrm{m})$, the down-regulation of Bcl-2 and the activation of caspases. Arsenic trioxide-treated cells were shown to manifest DNA damage and cell cycle arrest at the $\mathrm{G} 0$ or $\mathrm{G} 2 / \mathrm{M}$ phases.

Imatinib mesylate $\left(\right.$ Gleevec $^{\circledR}$, STI-571) is a selective inhibitor of Bcr-Abl tyrosine kinase, as well as other tyrosine kinases, such as the PDGF receptor (PDGFR) and c-Kit (2). STI-571 is now employed for the treatment of chronic myeloid leukemia and gastrointestinal stromal tumors, and its potential use in the treatment of other c-Kit-positive malignancies, including seminoma and acute myeloid leukemia, is currently being investigated (3). Many neuroblastoma cell lines and neuroblastoma tissue samples have been found to express PDGFR and c-Kit. Furthermore, STI-571 has been reported to inhibit the growth of neuroblastoma cells both in vitro and in vivo, via the suppression of PDGFR and c-Kit phosphorylation (4).

Since arsenic trioxide and STI-571 have been identified as good candidates for the treatment of neuroblastoma, we aimed to determine the effects of STI-571 with arsenic treatment on the growth of neuroblastoma cells in vitro, in relation to the state of MYCN amplification and expression, using SH-SY5Y, SK-N-SH and SK-N-BE(2) cells.

\section{Materials and methods}

Cell culture and chemicals. Imatinib mesylate (Gleevec, STI-571), which was generously provided by Novartis (Basel, Switzerland), was prepared as a $10-\mathrm{mM}$ stock solution in a sterile solution in DMSO. Neuroblastoma cell lines, SH-SY5Y (CRL-2266), SK-N-SH (HTB-11) and SK-N-BE(2) (CRL-2271) (all from ATCC, Manassas, VA, USA), as well as K562 cells, were cultured in RPMI-1640 media supplemented with heat-inactivated $10 \%$ FBS and antibiotics. 

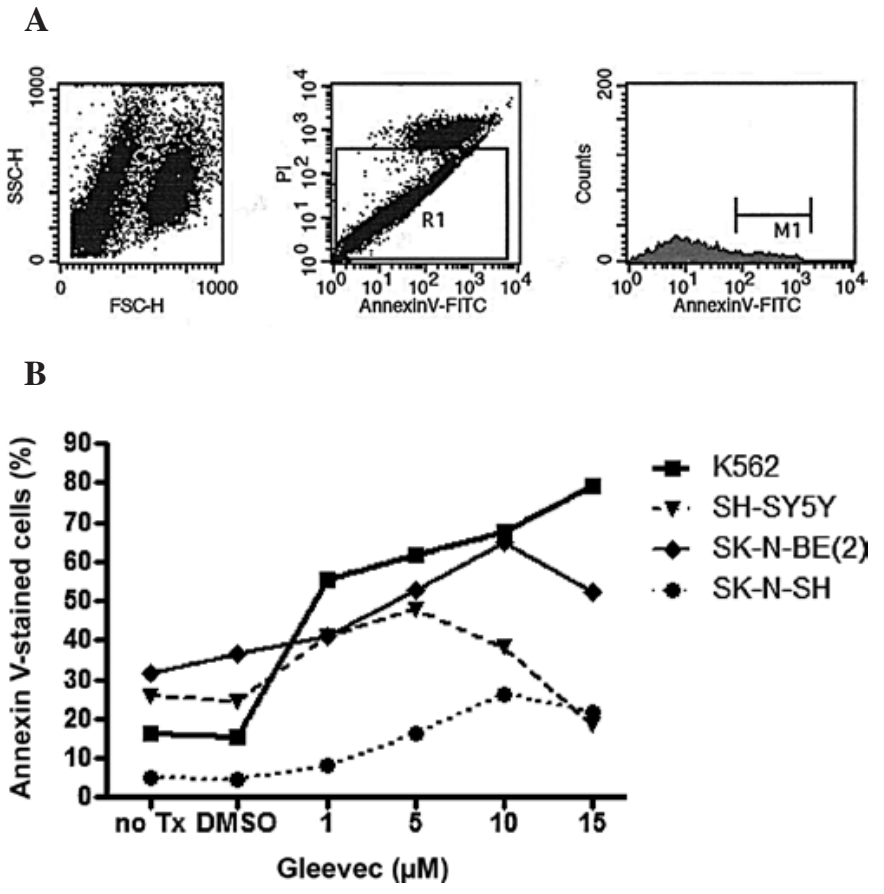

Figure 1. Annexin V staining for the detection of apoptotic cells. Cells were exposed to the indicated concentrations of STI-571 (Gleevec) for $72 \mathrm{~h}$. (A) The proportion of propidium iodide (PI)-negative cells (R1-gated), excluding the dead cells, is shown. (B) The percentage of Annexin V-positive (M1-gated) cells was calculated, and the percentage of apoptotic cells was compared to that in the untreated cell samples.

Proliferation and apoptosis assays. The cells $\left(5 \times 10^{5}\right.$ cells/ well) were treated for $72 \mathrm{~h}$ with arsenic trioxide, then detached with trypsin, washed twice in cold PBS, and resuspended in 1X Binding Buffer (10 mM HEPES, pH 7.4, 140 mM NaCl, $2.5 \mathrm{mM} \mathrm{CaCl}_{2}$ ) at a concentration of $1 \times 10^{6}$ cells $/ \mathrm{ml}$. The cells were then stained with Annexin $\mathrm{V}$ and propidium iodide (PI). After incubation for $15 \mathrm{~min}$ at room termperature in the dark, the cells were analyzed using the FACSCalibur system (BD, San Jose, CA, USA) with CellQuest software (BD).

For the 3-(4,5-dimethylthizol-2-yl)2,5-diphenyl tetrazolium bromide (MTT, M5655; Sigma) assay, the cells were plated into 96-well microtiter plates at a density of $5 \times 10^{3} / 150 \mu \mathrm{l}$ in fresh medium and treated with arsenic trioxide and/or STI-571. After $72 \mathrm{~h}, 20 \mu \mathrm{l}$ of MTT (5 mg/ml in PBS) was added to each well, and the plates were returned to the incubator for an additional $4 \mathrm{~h}$. At the end of the incubation period, the supernatants were discarded by suction and $200 \mu \mathrm{l}$ of DMSO was added to all wells in order to dissolve the dark blue formazan crystals. The plates were then covered with aluminum foil and gently agitated, then the absorbance was read at a wavelength of $570 \mathrm{~nm}$.

Immunoblots. The cells were lysed in a lysis buffer containing $150 \mathrm{mM} \mathrm{NaCl}, 1 \% \mathrm{NP}-40,0.5 \%$ DOC, $0.1 \%$ SDS, $50 \mathrm{mM}$ Tris- $\mathrm{Cl}$ ( $\mathrm{pH} 7.5$ ), $2 \mathrm{mM}$ sodium orthovanadate, $20 \mu \mathrm{g}$ / $\mathrm{ml}$ phenylmethylsulfonyl fluoride (PMSF) and $2 \mu \mathrm{g} / \mathrm{ml}$ of aprotinin. The lysates were sonicated with an ultrasonic homogenizer (Cole-Parmer, Vernon Hills, IL, USA) and then centrifuged for $10 \mathrm{~min}$ at $11,000 \mathrm{rpm}$ at $4^{\circ} \mathrm{C}$. The clear lysates were collected, and the protein concentrations of the lysates were determined using BCA kits (Pierce Chemical Co., Rockford, IL, USA). The protein was then loaded at $5 \mu \mathrm{g}$ per lane on a $10 \%$ SDS-PAGE gel. The antibodies used were antiMYCN Ab (\#9405; Cell Signaling Technology) and $\beta$-actin $\mathrm{Ab}$ (Sigma). Secondary antibodies were purchased from BioRad (Hercules, CA, USA).

Small interfering RNA (siRNA) transfection. siRNA against MYCN (siMYCN; siGENOME SMARTpool M-003913-010005) was purchased from Dharmacon, Inc. (Lafayette, CO, USA) (5), and the non-targeting control was obtained from Bioneer (Daejon, Korea). Transfection was conducted with Lipofectamine 2000 (Invitrogen, Carlsbad, CA, USA) in accordance with the manufacturer's recommendations. The cells were seeded on 96-well plates for the MTT assay and on 6-well plates for RNA preparation.

$R T$-PCR. Total RNA was extracted from siRNA-transfected cells using TRIzol reagent (Invitrogen), and RT-PCR was conducted for the confirmation of gene expression in the cell lines. The primer sequences were as follows: MYCN forward, 5'-ACC ACA AGG CCC TCA GTA CC-3' and reverse, 5'-GTG CAT CCT CAC TCT CCA CG-3'; GAPDH forward 5'-GTC TTC TCC ACC ATG GAG AA-3' and reverse 5'-CAT GCC AGT GAG CTT CCC GTT CA-3'. The PCR conditions were as follows: denaturation for $5 \mathrm{~min}$ at $94^{\circ} \mathrm{C}$, amplification for 30 cycles with $1 \mathrm{~min}$ at $94^{\circ} \mathrm{C}, 1 \mathrm{~min}$ at $55^{\circ} \mathrm{C}$ and a final extension for $1 \mathrm{~min}$ at $72^{\circ} \mathrm{C}$.

\section{Results}

Cellular apoptosis induced in neuroblastoma cells upon STI-571 treatment. Initially, we assessed the effects of STI-571 on three neuroblastoma cell lines: SK-N-BE(2), which has an MYCN amplication and expresses the N-Myc protein; SH-SY5Y, which expresses the N-Myc protein, but does not have an MYCN amplification; and SK-N-SH, which does not express N-Myc protein or have an MYCN amplification (6,7). K562 cells, which have a bcr-abl translocation, were used as a positive control. The percentage of Annexin $\mathrm{V}$-positive neuroblastoma cells was increased in the cell lines treated with concentrations $>1 \mu \mathrm{M}$ STI-571 (Fig. 1). Notably, the percentage of Annexin V-positive cells was increased to a greater extent in the SK-N-SH cells than in the SH-SY5Y or SK-N-BE(2) cells. Additionally, the percentage of Annexin $\mathrm{V}$-positive cells decreased at STI-571 concentrations of 10 and $15 \mu \mathrm{M}$ in the neuroblastoma cell lines. This was attributed to the removal of the dead cell fraction occurring during the washing procedure.

Cytotoxic effects induced in neuroblastoma cells after arsenic trioxide and STI-571 treatment. An MTT assay was conducted to measure the effects of arsenic trioxide and/or STI-571 treatment on cell proliferation in the neuroblastoma cell lines. As shown in Fig. 2, in the SK-N-SH and SH-SY5Y cell lines, treatment with $10 \mu \mathrm{M}$ of STI-571 suppressed proliferation, while this effect was not noted in the SK-N-BE(2) cells. Arsenic trioxide also induced a reduction in cell proliferation at 0.5 and $5 \mu \mathrm{M}$. In addition, treatment with STI-571 and arsenic trioxide exerted an additive or synergistic effect 


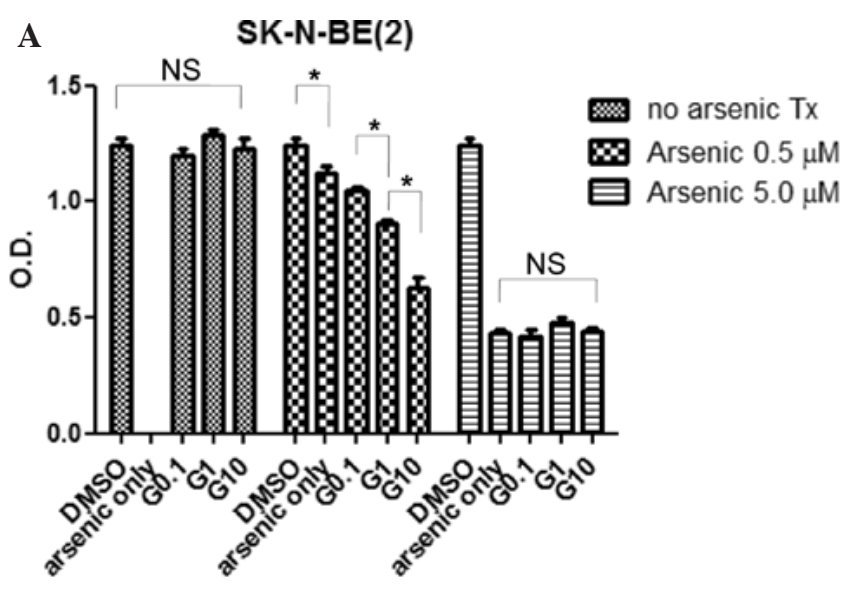

$\mathbf{B}$

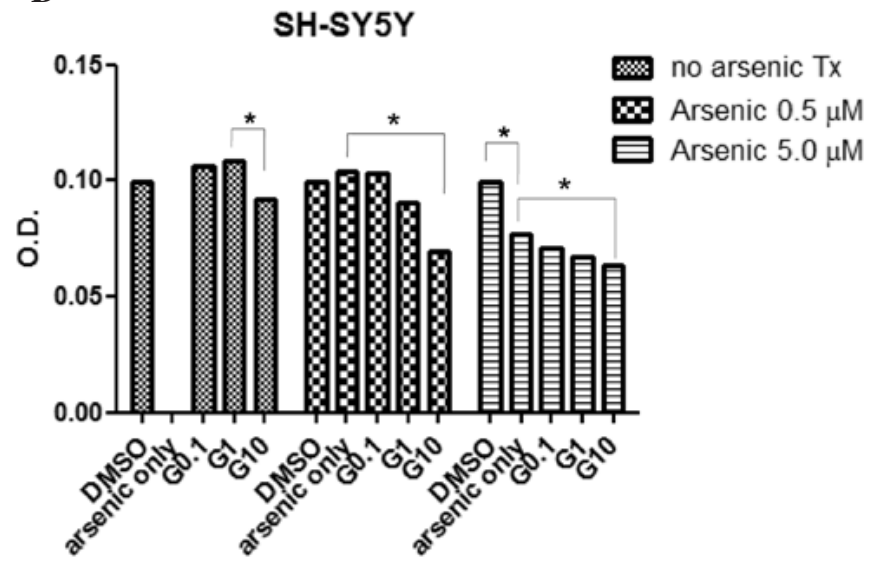

C

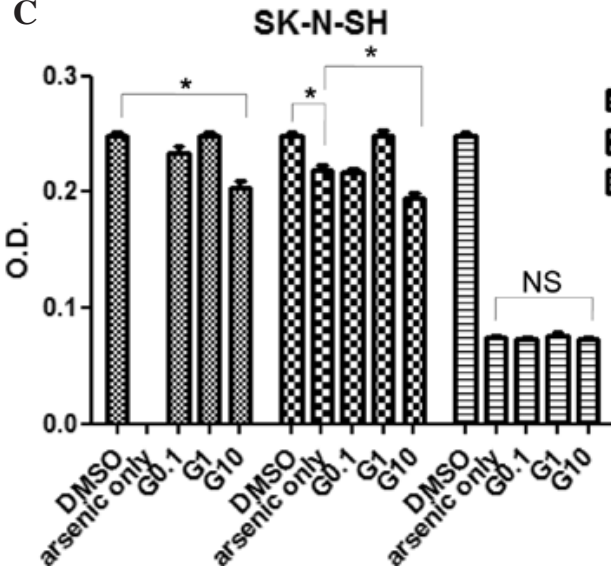

Figure 2. STI-571 and arsenic trioxide treatment inhibited the proliferation of neuroblastoma cells. (A) SK-N-BE(2), (B) SH-SY5Y and (C) SK-N-SH cells were plated at a density of $5 \times 10^{3} / 150 \mu \mathrm{l}$ in fresh medium and treated with arsenic trioxide and/or STI-571 (G) as indicated. After $72 \mathrm{~h}, 20 \mu \mathrm{l}$ of MTT (5 $\mathrm{mg} / \mathrm{ml}$ in PBS) was added to each of the wells. After $4 \mathrm{~h}$, dark blue formazan crystals were dissolved, and the absorbance of the plates was read at a wavelength of $570 \mathrm{~nm}$. Data are expressed in terms of optical density (O.D.) and expressed as the means $\pm \mathrm{SE}(\mathrm{n}=4)$. ${ }^{*} \mathrm{p}<0.05$; NS, not statistically significant.

on the inhibition of cell proliferation. Upon arsenic treatment at a concentration of $5 \mu \mathrm{M}$, proliferation was inhibited independent of the STI-571 concentration in the SK-N-BE(2) and SH-SY5Y cell lines. In the SH-SY5Y cells, in the presence of arsenic trioxide $(0.5$ and $5 \mu \mathrm{M})$, STI-571 exerted marked effects on cellular proliferation in a dose-dependent manner.
A

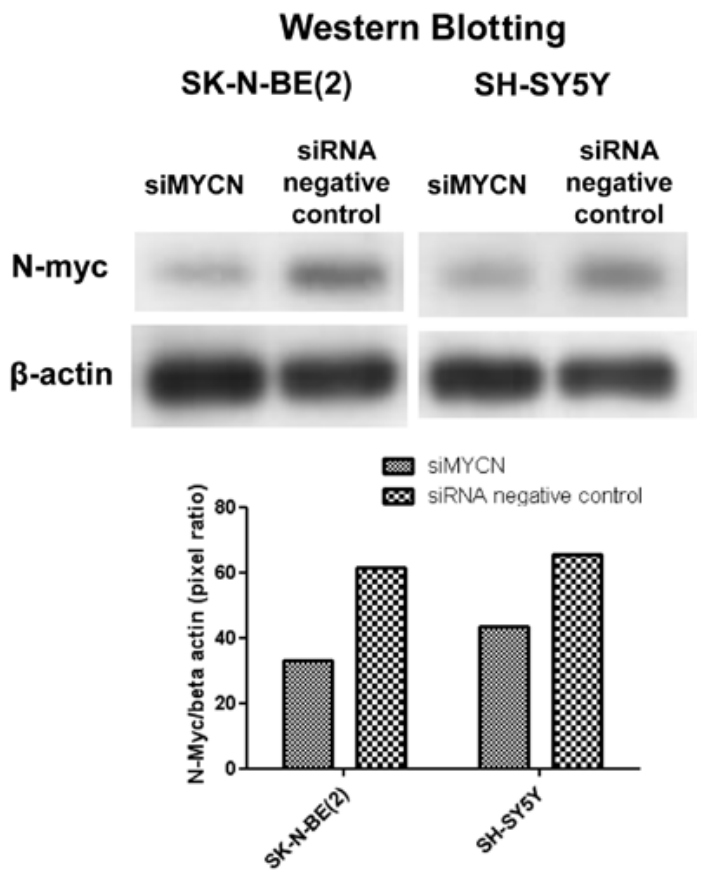

B

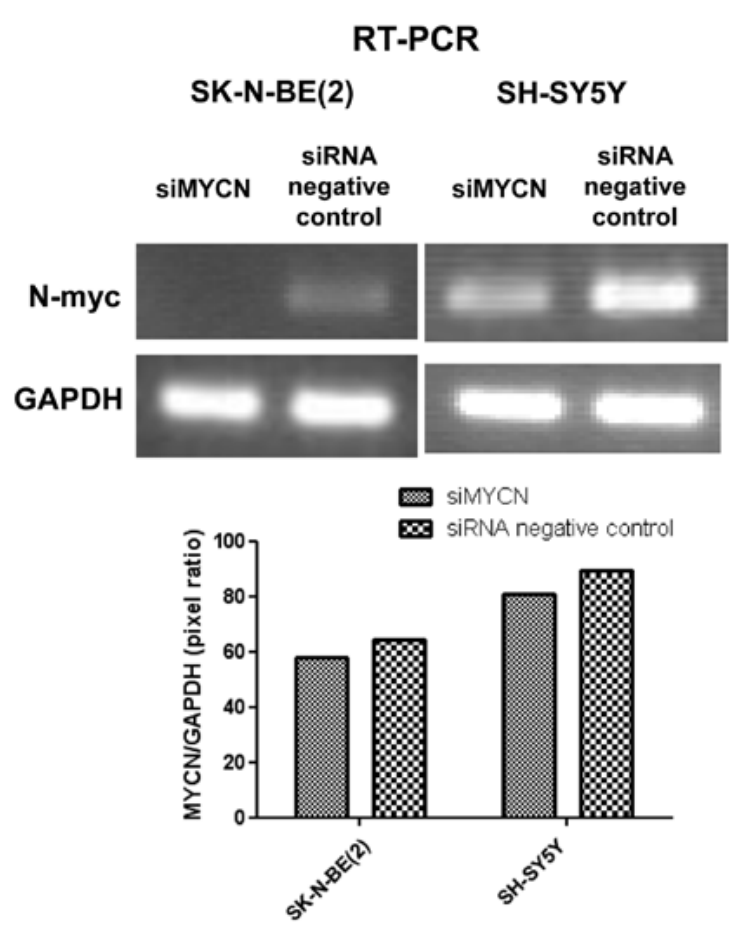

Figure 3. Knockdown of N-myc expression using siRNA in MYCNamplified/expressing SK-N-BE(2) and MYCN-expressing SH-SY5Y neuroblastoma cell lines. (A) Western blot analysis of $\mathrm{N}$-myc protein expression in neuroblastoma cells after $72 \mathrm{~h}$ of transfection. $\beta$-actin was used for the equal protein loading of the cell lysates. (B) Semi-quantitative RT-PCR analysis of MYCN mRNA expression in neuroblastoma cells after $72 \mathrm{~h}$ of transfection. GAPDH was used as a loading control. Data are expressed as a ratio of arbitary pixel density units.

Expression of N-myc knocked down by siRNA. Since the SK-N$\mathrm{BE}(2)$ and SH-SY5Y cell lines have been reported to express $\mathrm{N}$-myc, the differences noted in sensitivity (Fig. 1) to STI-571 may be related to $\mathrm{N}$-myc expression in neuroblastoma cells. Thus, we aimed to characterize the effects of N-myc knockdown on STI-571 and/or arsenic trioxide treatment. As shown 
A SK-N-BE(2)
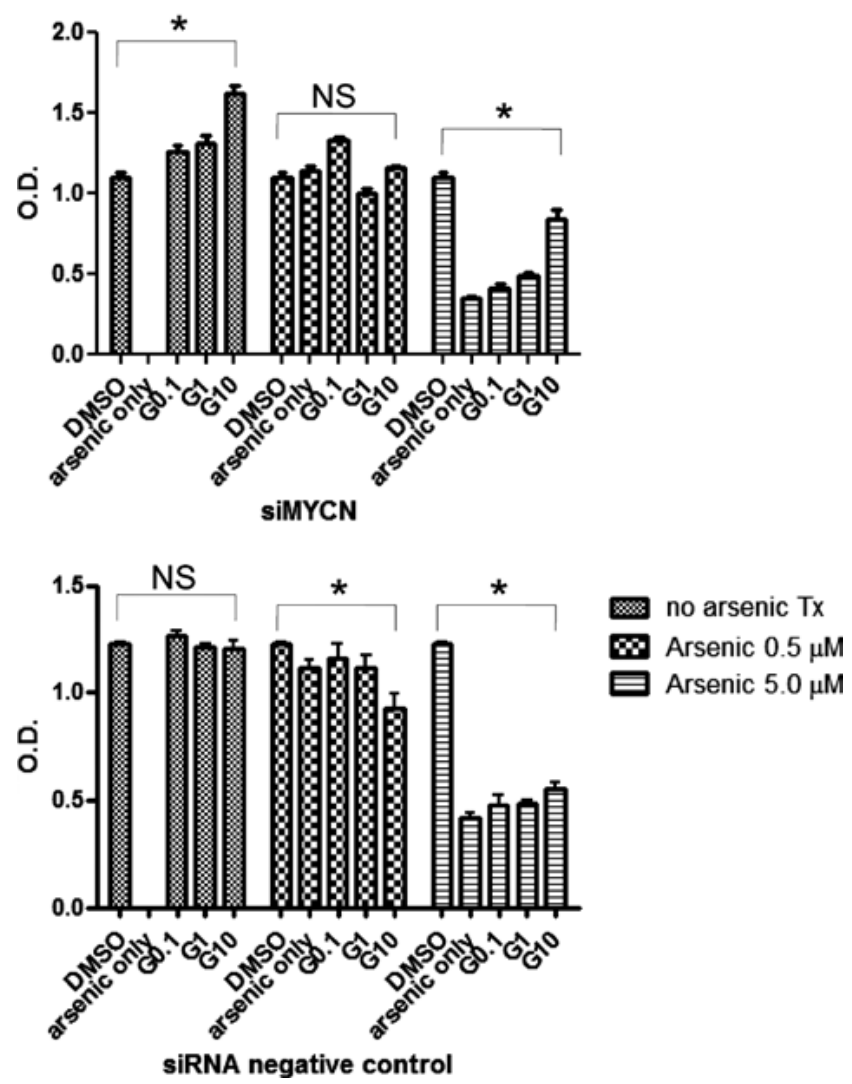

B
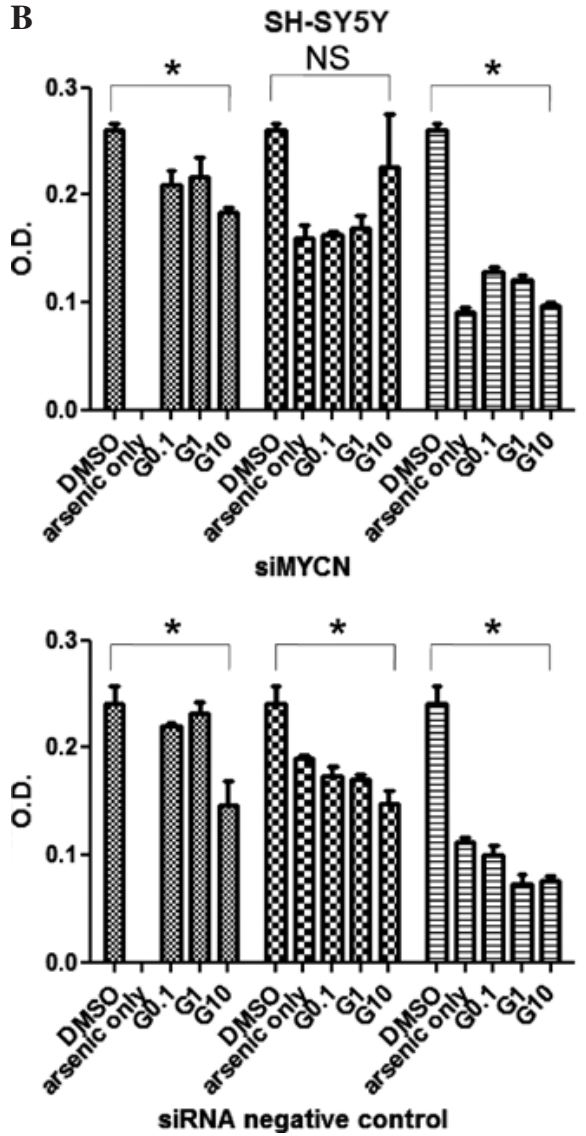

Figure 4. MYCN knockdown by RNAi abrogates the effects of STI-571 in neuroblastoma cell lines. (A) SK-N-BE(2) and (B) SH-SY5Y cells were plated at a density of $5 \times 10^{3} / 150 \mu \mathrm{l}$ in fresh medium and transfected with siMYCN or the negative siRNA duplex followed by exposure to arsenic trioxide and/or STI-571 (G) as indicated. After $72 \mathrm{~h}, 20 \mu \mathrm{l}$ of MTT $(5 \mathrm{mg} / \mathrm{ml}$ in PBS) was added to each well. After $4 \mathrm{~h}$, dark blue formazan crystals were dissolved, and the absorbance of the plates was read at a wavelength of $570 \mathrm{~nm}$. Data are shown in terms of optical density (O.D.) and expressed as the means \pm SE ( $=4)$. ${ }^{*} \mathrm{p}<0.05 ; \mathrm{NS}$, not statistically significant.

in Fig. 2, STI-571 treatment applied to the SK-N-BE(2) cell line manifesting an MYCN amplification had minimal influence on the suppressive effect. However, in SH-SY5Y cells, a dosedependent reduction in cell proliferation was observed, even at a concentration of $5 \mu \mathrm{M}$ of arsenic trioxide. Using siRNA against MYCN (siMYCN) as well as the control (negative), mRNA and protein expression was inhibited after siRNA treatment (Fig. 3).

Cytotoxic effects reversed in neuroblastoma cells after siMYCN treatment. In order to observe the effects of N-myc knockdown on arsenic trioxide and STI-571 treatments, the cells were transfected with siMYNC or siRNA negative controls and analyzed using an MTT assay. The siRNA negative control-transfected cells exhibited a higher sensitivity to STI-571 treatment than the siMYCN-transfected cells (Fig. 4). This suggests that N-myc knockdown does not render neuroblastoma cells more vulnerable to STI-571 treatment.

\section{Discussion}

In the present study, we found that treatment with STI-571 and arsenic trioxide exerted synergistic cytotoxic effects on three neuroblastoma cell lines: SH-SY5Y, SK-N-SH and $\mathrm{SK}-\mathrm{N}-\mathrm{BE}(2)$. In the MYCN-expressing/amplified cell lines, gene knockdown of MYCN via the siRNA method inhibited the therapeutic effects of STI-571 exposure.
MYCN amplification occurs in approximately $25 \%$ of neuroblastoma cases and has been profoundly correlated with cancer progression and treatment failure. Myc protein is a member of the family of basic-helix-loop-helix-leucine zipper transcription factors, and the increased expression of this protein results in the entry of cells into the cell cycle. The amplification and overexpression of MYCN in neuroblastoma have been correlated with treatment failure and a poor prognosis. As MYCN-expressing cancer cells often exhibit defects in the apoptotic pathway, the inhibition of MYCN expression may prove to be a useful target for the treatment of neuroblastoma. In pursuit of such targets, RNA interference may be employed for the knockdown of specific proteins in target cells. Therefore, the siRNA technique targeting MYCN was recently highlighted as a potential new therapeutic modality for the treatment of aggressive neuroblastoma (5).

However, it has also been reported that MYCN expression may increase the antitumor effect of various drug therapies, as MYCN expression was found to induce the transition from the G1-S to G2/M phase of the cell cycle (8). In addition, in K562 cells, c-Myc levels were not correlated with cell proliferation and c-Myc down-regulation was correlated with STI-571 activity, but not with STI-571-induced apoptosis (9). Therefore, in the present study we evaluated the effects of $\mathrm{N}$-myc knockdown by siRNA in combination with STI-571 and arsenic trioxide treatment, as both have been suggested as 
promising new therapeutic agents for the treatment of aggressive neuroblastomas.

STI-571 treatment applied to neuroblastoma cells was found to target c-Kit and PDGFR- $\alpha$ and $-\beta$ (10). In particular, $\mathrm{c}-\mathrm{Kit}$ and its ligand stem cell factor were reported to be expressed in neuroblastoma cells, as well as in primary tumors (3). Moreover, c-Kit has been reported to be preferentially expressed in MYCN-amplified neuroblastoma and was shown to inhibit STI-571-mediated proliferation (11).

In conclusion, STI-571 is a potential therapeutic drug for the treatment of neuroblastoma, particularly in MYCNamplified/expressing cancers. However, for the development of new therapeutic targets in cancer therapy, synergistic or possible antagonistic effects should be considered when using a combined therapeutic regimen.

\section{Acknowledgements}

This study was supported by the Korea Research Foundation Grant funded by the Korean Government (MOEHRD, Basic Research Promotion Fund) (KRF-2005-003-E00134).

\section{References}

1. Woo SY, Lee MY, Jung YJ, Yoo ES, Seoh JY, Shin HY, Ahn HS and Ryu KH: Arsenic trioxide inhibits cell growth in SH-SY5Y and SK-N-AS neuroblastoma cell lines by a different mechanism. Pediatr Hematol Oncol 23: 231-243, 2006.
2. Krause DS and van Etten RA: Tyrosine kinases as targets for cancer therapy. N Engl J Med 353: 172-187, 2005.

3. Mauro MJ, O'Dwyer M, Heinrich MC and Druker BJ: STI571: a paradigm of new agents for cancer therapeutics. J Clin Oncol 20: 325-334, 2002.

4. Beppu K, Jaboine J, Merchant MS, Mackall CL and Thiele CJ: Effect of imatinib mesylate on neuroblastoma tumorigenesis and vascular endothelial growth factor expression. J Natl Cancer Inst 96: 46-55, 2004.

5. Kang JH, Rychahou PG, Ishola TA, Qiao J, Evers BM and Chung DH: MYCN silencing induces differentiation and apoptosis in human neuroblastoma cells. Biochem Biophys Res Commun 351: 192-197, 2006.

6. Brodeur GM, Green AA, Hayes FA, Williams KJ, Williams DL and Tsiatis AA: Cytogenetic features of human neuroblastomas and cell lines. Cancer Res 41: 4678-4686, 1981.

7. Gilbert F, Feder M, Balaban G, Brangman D, Lurie DK, Podolsky R, Rinaldt V, Vinikoor N and Weisband J: Human neuroblastomas and abnormalities of chromosomes 1 and 17. Cancer Res 44: 5444-5449, 1984.

8. Paffhausen T, Schwab M and Westermann F: Targeted MYCN expression affects cytotoxic potential of chemotherapeutic drugs in neuroblastoma cells. Cancer Lett 250: 17-24, 2007.

9. Gomez-Casares MT, Vaque JP, Lemes A, Molero T, Delgado MD and Leon J: C-myc expression in cell lines derived from chronic myeloid leukemia. Haematologica 89: 241-243, 2004.

10. Buchdunger E, Cioffi CL, Law N, Stover D, Ohno-Jones S, Druker BJ and Lydon NB: Abl protein-tyrosine kinase inhibitor STI571 inhibits in vitro signal transduction mediated by c-kit and platelet-derived growth factor receptors. J Pharmacol Exp Ther 295: 139-145, 2000.

11. Vitali R, Cesi V, Nicotra MR, McDowell HP, Donfrancesco A, Mannarino O, Natali PG, Raschella G and Dominici C: c-Kit is preferentially expressed in MYCN-amplified neuroblastoma and its effect on cell proliferation is inhibited in vitro by STI-571. Int J Cancer 106: 147-152, 2003. 